\title{
DISCUSSION OF THE MEAN SQUARE ERROR OF PREDICTION IN THE CHAIN LADDER RESERVING METHOD
}

\author{
BY
}

\section{GARY G. VENTER}

\section{Chain Ladder Variance Formula}

For a dozen or so years the chain ladder models of Murphy and Mack have been known to give the same reserve estimates but differ slightly in their assumptions and in their calculations of the reserve variance. Up until now no one had provided a detailed analysis of the source of the difference in variance. The current (BBMW) paper shows that this arises from a slight difference in the estimates of the parameter variance piece, and this in turn comes from the fact that for Murphy the errors in the estimates of the development factors are independent, while under Mack's assumptions they are merely uncorrelated. This would suggest that any given data could be tested for the difference in assumptions by computing correlations of functions of the estimated factors. For instance a finding that some functions of the factors are correlated but the factors themselves are uncorrelated would support Mack's model over Murphy's.

Now the Mack-Quarg-Braun (MQB) discussion has short-cut this approach by showing that under the basic chain ladder assumptions of Mack or BBMW, the squares of consecutive development factors are negatively correlated. This would seem to rule out the independence assumption and Murphy's formula.

The BBMW model has the same formulas as Murphy's. However it is not entirely clear if they have the same problem with independence of factor estimates. Mack showed that the estimates are conditionally uncorrelated given the portion of the triangle available at the relevant points. BBMW introduce a resampling methodology which yields hypothetical new factors identical in distribution to the old ones and which are conditionally independent given the entire original triangle. (Conditional on the whole triangle the original factors are deterministic.) They then use this conditional independence to derive Murphy's variance result for the new factors. The result holds for the original factors because they have the same distribution as the new factors. Thus BBMW assert that they are not assuming independence. This is a novel and interesting approach but could use some more vetting to be sure it is valid. However independence of factors seems to creep in anyway, although as a conclusion rather than an assumption. MQB's result of non-independence of adjacent factor estimates seems to arise naturally from the denominator of one factor being almost the numerator of the next, and it is not clear that resampling avoids this. 
In fact Mack's original finding that the factor estimates are uncorrelated is somewhat surprising in this context.

\section{Applicability of Chain Ladder to the Example}

Many actuaries have interpreted the existence of models of the chain ladder as support for using it. A more nuanced view is that having a model provides a method for testing applicability of the chain ladder to a given data $\operatorname{set}^{1}$. BBMW note that they are not representing that either model applies to the data in their example. This leaves the applicability open. Some testing methodology is reviewed below and applied to the example development triangle. The assumptions of both of the chain ladder models are found to be violated by this data.

The models of Mack and Murphy-BBMW express the expected incremental loss at one lag as a factor times the cumulative loss at the previous lag. Whether or not this holds for a given loss triangle is easy to test by regression methods. The incremental loss at lag $d+1$ (call this $y$ ) can be estimated as a linear regression on the cumulative loss at lag $d$ (called $x$ ). If the model applies, the additive constant from the regression should not be significantly different from zero, but the factor should be. Because the variance is assumed to be proportional to $x$, both $y$ and $x$ should be divided by $x^{\frac{1}{2}}$ before doing the regression.

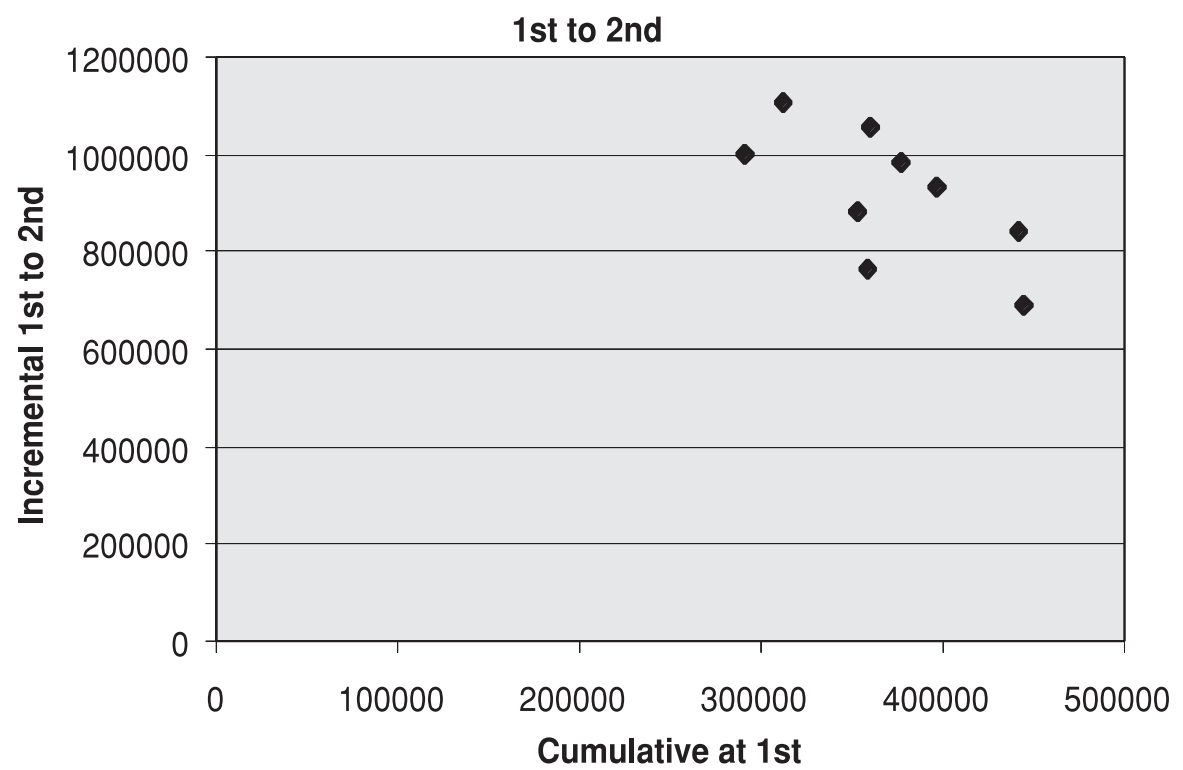

Figure 1.

1 See for instance Mack(1994), Venter (1998), Barnett and Zehnwirth (2000). 
The regressions were carried out for the first five columns of the sample array. The combination of factor significant and constant not fails for all of them. The only significant regression is in fact $1^{\text {st }}$ to $2^{\text {nd }}$, but the constant there is also significant, with higher losses in the first period predicting lower losses emerging in the second. The sample points are graphed in Figure 1. Points from a factor model would look like a straight line through the origin, with some randomness around it, which this does not.

Another assumption of the chain-ladder models is that the development factors are not correlated. This is violated for the $3^{\text {rd }}$ to $4^{\text {th }}$ and $4^{\text {th }}$ to $5^{\text {th }}$ factors, which are significantly negatively correlated. Independence of the factor estimates, which is required for Murphy-BBMW but not Mack, is also violated. This can be checked by looking at the correlation of squared deviations of the individual factors from the column mean factors. These are significantly positive for the $3^{\text {rd }}$ to $4^{\text {th }}$ factor with both the $2^{\text {nd }}$ to $3^{\text {rd }}$ and $4^{\text {th }}$ to $5^{\text {th }}$ factors.

The chain-ladder independence assumptions also imply that there are no calendar-year (diagonal) effects in the triangle. This implication can be violated for instance by accelerated or stalled claim department activity in a calendar year. Such a departure would often be made up for in a later year or years, so more than one diagonal can be affected. A similar pattern can arise from inflation operating on calendar years. Inflation operating on year of origin is built into the factor approach, as each year gets its own level. But there can appear to be inflation by year of origin that is actually generated by calendar year inflation. If the latter varies by year, a pattern of high and low residuals can show up by diagonal. A large variation in residuals among diagonals could suggest that either calendar year inflation or claim department instability is affecting the data.

Table 1 shows the average residual and fraction of positive residuals for the last 3 diagonals of the example. Only the second to last is comfortably random, suggesting that there are diagonal effects that have not been picked up by the model.

TABLE 1

\begin{tabular}{c|c}
\hline \hline Average residual & Fraction positive \\
\hline$(160,758)$ & 1 of 7 \\
22,930 & 3 of 8 \\
74,814 & 6 of 8 \\
\hline \hline
\end{tabular}

\section{Multiplicative Fixed-Effects Model Comparison}

The differences among the chain-ladder variances discussed so far are relatively minor. There is a quite different model which gives the same reserves as the chain-ladder but different fitted values and a different variance. Instead of 
an incremental cell's expected losses being a factor times the previous cumulative, it is a percentage of ultimate losses. E.g., in a paid loss triangle expected paids follow a payout pattern based on ultimate losses even though the ultimate is not known.

Each cell is modeled as a random innovation plus the product of a row and column parameter (e.g., ultimate and fraction paid), so this can be called a multiplicative fixed-effects model. It agrees with the chain-ladder estimates when losses follow an aggregate distribution consisting of a Poisson frequency and a Constant Severity (PCS). That is, each cell of the incremental triangle has a Poisson loss frequency and all cells have the same constant loss severity. This is of course a gross over-simplification of the loss generation process, but nevertheless it might give a reasonable account of the statistical behavior of some loss triangles. Denoting $b$ as the constant severity, and $x$ as the aggregate loss for a cell, the distributional assumption is that $x / b$ is Poisson. The resulting aggregate loss distribution has also been called the over-dispersed Poisson. It has mean $b \lambda$ and variance $b^{2} \lambda$, and so variance-to-mean ratio $b$, which does not have to be above 1 .

The interest here is that if the row and column factors are estimated by maximum likelihood, then the reserve calculation is the chain-ladder algorithm. For the pure Poisson case this was shown by Hachemeister and Stanard (1975) although it was not published formally until Kremer (1985) in German and Mack (1991) in English. The extension to the PCS model was by Renshaw and Verrall (1998). A good presentation is Clark (2003), who assumes a parameterized distribution for the payout pattern, but a discrete distribution is a special case. He also discusses the Cape Cod model, which is PCS with all accident years at the same level. None of the cited papers compare the resulting variance to that from Mack or Murphy, however.

The PCS model estimates the expected value of the observed cells of the triangle by the last diagonal backed down with the development factors. From the chain ladder viewpoint this is predicting past losses from later information. But the PCS is not the chain ladder. The paradigm of loss emergence as a fixed payout pattern can be traced back at least to the Bornheutter-Ferguson (BF) model, so PCS is more like a parameterized version of BF than of the chain ladder.

Backing down from the latest diagonal often gives better predictions of observed incremental losses, and so may give an advantage in goodness of fit to the PCS. But this fit has to be penalized due to the extra parameters. It is actually not easy to compare fits, because the chain-ladder models are conditional given the triangle, whereas PCS uses the data in the triangle to fit unobserved parameters unconditionally. Perhaps the best way to compare the fits is to calculate the variance of the estimated reserves for each model, including parameter uncertainty, and interpret the model with the lower variance as having the best fit. This credits the PCS if it has the better fit but penalizes it for having more parameters.

Clark shows how to measure the PCS variance with the information matrix from MLE and the delta method, which gives the parameter variance for a 


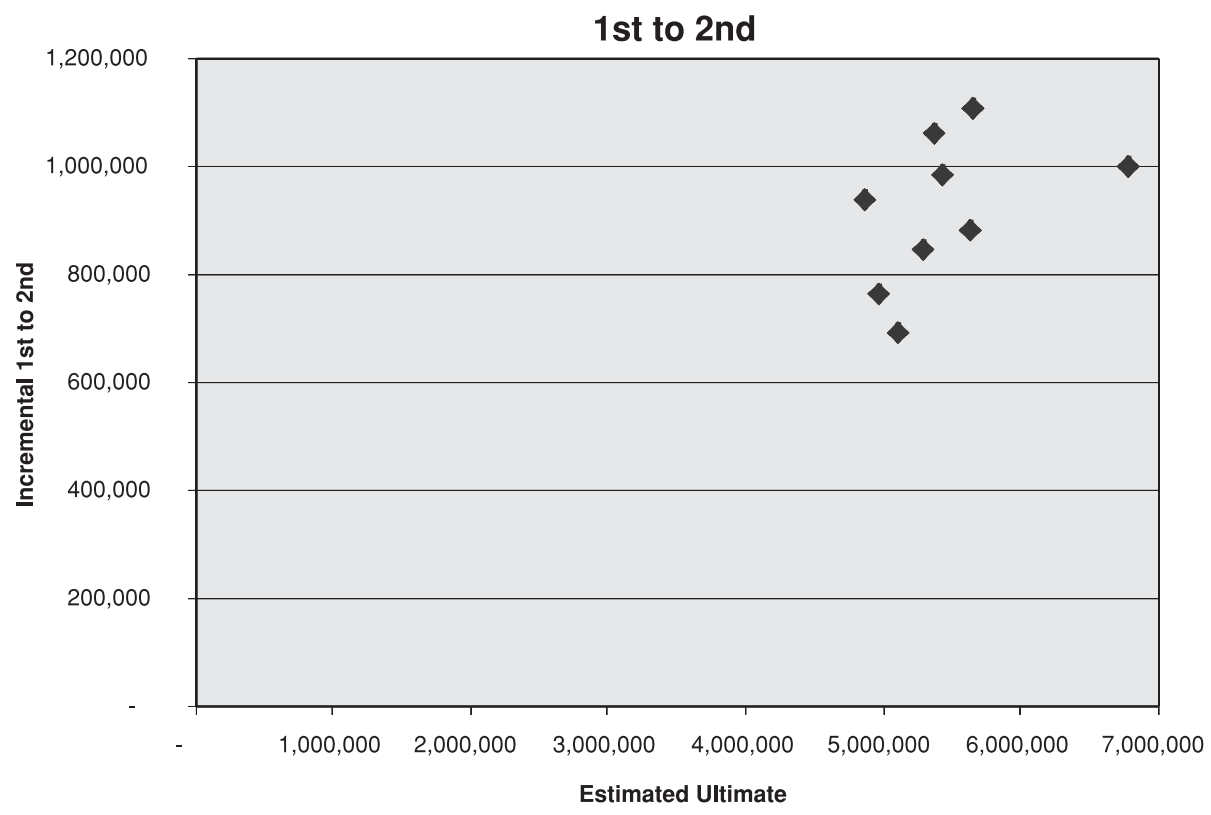

Figure 2.

function of MLE-estimated parameters. In this case the function is the estimated reserve. For the triangle in the BBMW paper, the PCS prediction standard error can be shown to be 2,827,000 compared to 2,447,000 for Mack's model. Here the better fit from backing down from the latest diagonal does not compensate for the extra parameter variance of the PCS. The process standard deviation reduced by almost $50 \%$, but the increase in the parameter standard deviation was nearly $70 \%$.

Of course the PCS itself has assumptions that need to be checked. The analogue to comparing incremental losses to previous cumulative is to compare them to estimated ultimate, because PCS postulates that expected incrementals are factors times ultimate losses. Figure 2 graphs the $1^{\text {st }}$ to $2^{\text {nd }}$ incrementals vs. expected ultimate. This looks more plausible as a straight line through the origin than were the same incrementals as a function of losses at $1^{\text {st }}$, shown in Figure 1.

The PCS model has other key assumptions, like constant variance-to-mean ratio and no diagonal effects. Without going into detail, the constant ratio has support in this triangle, but there is still a problem with diagonal effects. The PCS actually assumes all cells are independent, so any correlation of any functions of rows, columns, or diagonals would be problematic. As noted above, some such were found for this triangle, so the PCS model assumptions are also violated. 


\section{Conclusions}

The BBMW paper provides some clarity as to why the Mack and Murphy models have different variance results, coming down to the issue of independent vs. uncorrelated factor estimates. However the work of the MQB reviewers supports non-correlation but not independence, and so the Mack formula. Neither the Mack nor Murphy-BBMW chain ladder assumptions are met by the data in the example triangle. The PCS multiplicative fixed-effects model gives the same reserve estimate and provides a significantly better fit but a higher variance estimate for this data, and its assumptions are not satisfied either. There are numerous other models in the literature that could be tried. However the negative correlation of the $3^{\text {rd }}$ and $4^{\text {th }}$ developments could be a problem for many of them.

\section{REFERENCES}

Barnett, G. and Zehnwirth, B. (2000) Best Estimates for Reserves, CAS Proceedings, 245-321.

Clark, D.R. (2003) LDF Curve-Fitting and Stochastic Reserving: A Maximum Likelihood Approach. CAS Forum (Fall): 41-92.

Hachemeister, C. and Stanard, J. (1975) IBNR Claims Count Estimation With Static Lag Functions. Presented at 1975 ASTIN Colloquium, Portimao, Portugal.

Kremer, E. (1985) Einfuhrung in die Versicherungsmathematik, Vandenhoek \& Ruprecht, Gottingen.

MACK, T. (1991) A simple parametric model for rating automobile insurance or estimating IBNR claims reserves. ASTIN Bulletin 21(1), 93-109.

MACK, T. (1994) Measuring the Variability of Chain Ladder Reserve Estimates, CAS Forum (Spring): 101-182.

Murphy, D. (1994) Unbiased Loss Development Factors, CAS Proceedings LXXXI: 154-222.

Renshaw, A. and Verrall, R.J. (1998) A Stochastic Model Underlying the Chain Ladder Technique. British Actuarial Journal 4, 903-23.

Venter, G. (1998) Testing the Assumptions of Age-to-Age Factors. CAS Proceedings, LXXXV: $807-47$. 